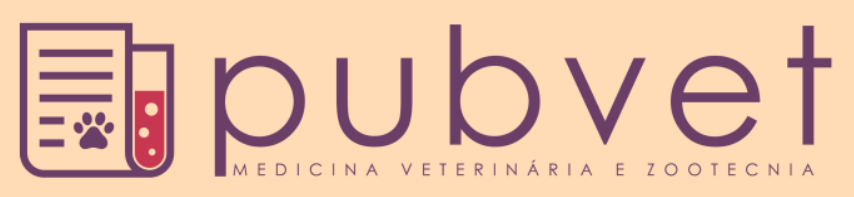

https://doi.org/10.22256/pubvet.v12n1a3.1-5

\title{
Estimativa de correlações fenotípicas de características visuais e ultrassonográficas em carcaças bovinas da raça Nelore
}

\author{
Onofre Oliveira Moraes Neto ${ }^{\oplus} 1 *$, Rodrigo Zaiden Taveira ${ }^{\ominus 2}$, Ana Christina Sanches ${ }^{\ominus} 3$, \\ Ludmilla Costa Brunes 94
}

1Discente em Zootecnia pela Pontifícia Universidade Católica de Goiás. Goiânia, Goiás, Brasil. E-mail: neto.zoo@hotmail.com 2Professor da Pontifícia Universidade Católica de Goiás. Goiânia, Goiás, Brasil. E-mail: rodrigozaiden@gmail.com ${ }^{3}$ Professora da Pontifícia Universidade Católica de Goiás. Goiânia, Goiás, Brasil. E-mail: anachrissanches@hotmail.com

${ }^{4}$ Aluno de Doutorado em Zootecnia da Empresa Brasileira de Pesquisa Agropecuária-Arroz e Feijão, Goiás, Brasil.

*Autor para correspondência

RESUMO. O objetivo foi estimar correlações fenotípicas de características de rendimento e acabamento de carcaça bovina. Foram empregadas avaliações por meio de escores visuais e por ultrassonografia. Três características foram avaliadas utilizando-se os escores visuais, sendo: musculatura (1 a 6), tamanho (1 a 5) e profundidade (1 a 5). Pela ultrassonografia foram avaliadas as seguintes características: área de olho de lombo (AOL), espessura de gordura subcutânea (EGS) e espessura de gordura subcutânea na garupa (EGP8). Os animais foram mantidos em pastagens tropicais com e sem complementação alimentar. Considerando os animais mantidos sem complementação alimentar as estimativas de correlação obtidas da AOL com as características de musculatura, frame e profundidade foram $0,15,0,17$ e $-0,09$ respectivamente. As estimativas de correlações da EGS com as características de musculatura, frame e profundidade foram 0,22, 0,07 e 0,24, respectivamente. As estimativas de correlações da EGP8 com as características de musculatura, frame e profundidade foram $0,11,-0,04$ e 0,18 , respectivamente. Considerando os animais mantidos com complementação alimentar as estimativas de correlação obtidas da AOL com as características de musculatura, frame e profundidade foram $0,30,-0,06$ e 0,17 , respectivamente. As estimativas de correlações da EGS com as características de musculatura, frame e profundidade foram $0,24,0,01$ e 0,24, respectivamente. As estimativas de correlações da EGP8 com as características de musculatura, frame e profundidade foram $0,20,0,03$ e 0,14 , respectivamente.

Palavras chave: melhoramento genético, musculatura, precocidade

\section{Estimates of phenotypic correlations of visual and ultrasonographic carcass characteristics in Nellore breed}

ABSTRACT. The aim was estimate phenotypic correlations of characteristics for animal breeding for beef carcass. Therefore, we employed the method of visual scores and the ultrasound method. Three criteria in the visual scores method, where: musculature (1 to 6), frame (1 to 5) and depth (1 to 5). In the ultrasound method was captured images of live animal carcass. Thus, they were considered: the Rib Eye Area (REA), the backfat thickness (BF); ribs and fat thickness measured in croup (RF). The results were divided into management of tropical pastures with and without food supplementation. Correlation estimates obtained in the management of tropical pastures for REA were 0.15 (muscles), 0.17 (frame) and -0.09 (depth) for BF were 0.22, 0.07, 0.24 and for RF were 0.11, 0.04, 0.18 , respectively. Since the estimates obtained in the management of tropical pastures with 
food supplementation for REA were $0.30,-0.06,0.17$, for EG were $0.24,0.01,0.24$ and RF were $0.20,0.03$ and 0.14 , respectively.

Keywords: genetic improvement, precocity, rib eye area and the backfat thickness

\title{
Estimación de correlaciones fenotípicas de características visuales y ultrasonográficas en canales bovinas de raza Nelore
}

\begin{abstract}
RESUMEN. El objetivo fue estimar correlaciones fenotípicas de características de rendimiento y acabado de canal bovina. Se emplearon evaluaciones por medio de escores visuales y por ultrasonografía. Se evaluaron tres características utilizando los escores visuales, siendo: musculatura ( 1 a 6 ), tamaño (1 a 5) y profundidad (1 a 5). Por ultrasonido se evaluó: área de lomo (AOL), espesor de grasa subcutánea (EGS) y espesor de grasa subcutánea en la grupa (EGP8). Los animales se mantuvieron en pasturas tropicales con y sin complemento alimenticio. Teniendo en cuenta los animales mantenidos sin complemento alimenticio las estimaciones de correlación obtenidas de AOL con las características de musculatura, marco y profundidad fueron $0,15,0,17$ y $-0,09$ respectivamente. Las estimaciones de correlaciones de EGS con las características de musculatura, marco y profundidad fueron $0,22,0,07$ y 0,24 , respectivamente. Las estimaciones de correlaciones de la EGP8 con las características de musculatura, frame y profundidad fueron $0,11,-0,04$ y 0,18 , respectivamente. Los animales mantenidos con complementación alimenticia las estimaciones de correlación obtenidas de la AOL con las características de musculatura, marco y profundidad fueron $0,30,-0,06$ y 0,17 , respectivamente. Las estimaciones de correlaciones de EGS con las características de musculatura, marco y profundidad fueron $0,24,0,01$ y 0,24 , respectivamente. Las estimaciones de correlaciones de la EGP8 con las características de musculatura, marco y profundidad fueron $0,20,0,03$ y 0,14 , respectivamente.
\end{abstract}

Palabras claves: mejoramiento genético, musculatura, precocidad

\section{Introdução}

A avaliação visual de carcaça permite identificar animais com maior precocidade de terminação, melhor musculatura e conformação frigorífica (Yokoo et al., 2009). Com esse método, de acordo com Koury Filho et al. (2009), maiores números de animais podem ser avaliados sem que se precise submetê-los a mensurações, o que minimiza o estresse dos animais e torna o processo mais ágil e de menor custo. A busca por precocidade de acabamento é um assunto em evidência, pois, o encurtamento do ciclo de produção de animais criados a pasto é um grande objetivo da bovinocultura de corte brasileira. Para isso os escores visuais podem ser utilizados para seleção de fenótipos que atenda as exigências do mercado (Faria et al., 2007).

Apesar das estimativas de herdabilidade medianas para características de carcaça, avaliadas por ultrassonografia, como a área de olho de lombo, espessura de gordura subcutânea e espessura de gordura subcutânea na garupa, as mesmas podem ser utilizadas como critério de seleção para obtenção de progresso genético em rebanhos de bovinos (Faria et al., 2015).

Face ao exposto objetivou-se estimar correlações fenotípicas de características de rendimento e acabamento de carcaça bovina.

\section{Material e Métodos}

Foram mensurados e analisados 2 grupos de manejo, o primeiro com 181 animais e o segundo com 123 animais, na ocasião do sobreano, com peso médio de $373 \pm 45 \mathrm{~kg}$ e $459 \pm 37 \mathrm{~kg}$, respectivamente. Os animais do primeiro grupo permaneceram em pastagens de capim Brachiara brizantha sem complementação alimentar e os animais do segundo grupo foram mantidos em pastagens de capim Brachiara brizantha com complementação alimentar proteica.

Foram empregadas avaliações por meio de escores visuais e por ultrassonografia. Três características foram avaliadas utilizando-se os escores visuais, sendo: musculatura (MUSC) de 1 a 6, tamanho (FRAME) de 1 a 5 e 
profundidade (PROF) de 1 a $5 . \quad$ Pela ultrassonografia foram avaliadas a as seguintes características: área de olho de lombo (AOL), espessura de gordura subcutânea (EGS) e espessura de gordura subcutânea na garupa (EGP8). Para avaliação da musculosidade foram verificados dois pontos no animal, sendo: o perímetro do antebraço e soldra. As notas atribuídas variaram de 1 a 6 e quanto maior a nota, melhor. Em relação ao tamanho as notas variaram de 1 a 5 , sendo os maiores animais possuidores de maiores notas. $\mathrm{Na}$ avaliação da profundidade as notas variaram de 1 a 5 e quanto maior a nota melhor.

Para a mensuração das características de carcaça, foram coletadas imagens de ultrassonografia por meio de um aparelho ALOKA 500V, com sonda linear de $17,2 \mathrm{~cm}$, de 3,5 MHz e um acoplador acústico em conjunto com um sistema de captura de imagens (Blackbox, Bionitrics, Inc., Ames, IA, EUA). As características avaliadas foram área de olho de lombo (AOL), espessura de gordura subcutânea (EGS), medidas entre as $12^{\mathrm{a}}$ e a $13^{\mathrm{a}}$ costelas, no músculo Longissimus dorsi e a gordura subcutânea sobre o músculo Biceps femoris e Gluteus medius (EGP8).

As análises estatísticas foram realizadas pelo programa estatístico R. As correlações foram estimadas por meio da correlação de Pearson $(\mathrm{P}<0,01)$ e as médias comparadas pelo teste $\mathrm{t}(\mathrm{P}<0,01)$.

\section{Resultados e Discussão}

A Tabela 1 dada abaixo apresenta a estatística descritiva referente às avaliações de carcaça dos animais pertencentes ao grupo de manejo 1 e 2.

Tabela 1. Valores mínimos, médias, desvios-padrões, valores máximos e coeficientes de variação da área de olho de lombo e espessura de gordura de bovinos da raça Nelore criados em pastagens tropicais

\begin{tabular}{lccccccc}
\hline & \multicolumn{3}{c}{ Sem complementação alimentar } & & \multicolumn{3}{c}{ Com complementação alimentar } \\
\cline { 2 - 3 } Variáveis & $\begin{array}{c}\text { Área de olho de } \\
\text { lombo, } \mathrm{cm}^{2}\end{array}$ & $\begin{array}{c}\text { Gordura } \\
\text { subcutânea, } \\
\text { mm }\end{array}$ & $\begin{array}{c}\text { Gordura } \\
\text { subcutânea na } \\
\text { garupa, mm }\end{array}$ & & $\begin{array}{c}\text { Área de olho } \\
\text { de lombo, } \\
\mathrm{cm}^{2}\end{array}$ & $\begin{array}{c}\text { Gordura } \\
\text { subcutânea, mm }\end{array}$ & $\begin{array}{c}\text { Gordura } \\
\text { subcutânea na } \\
\text { garupa, mm }\end{array}$ \\
\hline Mínimo & 45,42 & 1,02 & 1,02 & & 51,29 & 1,02 & 1,02 \\
Média \pm DP & $57,72 \pm 5,59$ & $1,81 \pm 0,58$ & $2,36 \pm 0,86$ & & $69,75 \pm 6,35$ & $2,68 \pm 0,95$ & $3,61 \pm 1,32$ \\
Máximo & 81,42 & 5,08 & 5,33 & & 83,68 & 5,33 & 7,24 \\
CV $(\%)$ & 9,68 & 32,04 & 36,44 & & 9,10 & 35,84 & 36,56 \\
\hline
\end{tabular}

As estimativas obtidas nas seguintes análises estatísticas para as características de avaliação de carcaça por ultrassonografia (Tabela 1) foram próximas da literatura publicada por Faria et al. (2009), Yokoo et al. (2009) e Faria et al. (2015), sendo que o grupo de contemporâneo obteve somente manejo alimentar a base de pastagens tropicais. Destacaram-se alguns animais que obtiveram máxima avaliação de AOL, EGS e EGP8 com respectivamente $81,4,5,1$ e 5,3. Animais em pastagens tropicais são de superioridade numérica em nosso rebanho efetivo nacional. Assim, é possível melhorar e produzir com eficiência a pasto. $\mathrm{O}$ grupo de contemporâneo com manejo alimentar a base de pastagens tropicais com complementação alimentar teve suas médias superiores às do grupo anterior, alguns animais obtiveram máxima avaliação de AOL, EGS e EGP8 com respectivamente 83,7, 5,3 e 7,2. Animais sujeitos a ambiência máxima que lhes permitem expressar seu potencial genético máximo, são de superioridade em rebanhos melhoradores.

As correlações obtidas para o grupo de manejo a base de pastagens tropicais (Tabela 2) estão em concordância com Yokoo et al. (2009) e foram significativas para a AOL com musculosidade e frame, o que nos faz pensar em boa correlação entre os métodos utilizados para tamanho e rendimento. Observou-se que EGS com musculosidade e profundidade, características que induzem precocidade e acabamento para animal superior. Observou-se que EGP8 com profundidade correlacionada com precocidade sexual.

O grupo de manejo alimentar a base de pastagens tropicais com complementação alimentar, as correlações foram próximas as de Cancian et al. (2014), Faria et al. (2015), Yokoo et al. (2009), sendo significativo AOL com musculosidade e profundidade, EGS com musculosidade e profundidade, EGP8 com 
musculosidade. Diferente do manejo anterior; porém na mesma sequência linear, animais submetidos à ambiência que lhes proporciona expressar seu potencial genético são mais precoces em ambas as características (Tabela 3), animais que atingem a extremidade de tamanho tanto para maior quanto para menor, são indesejados para este sistema.

Tabela 2. Estimativa de correlação, simples ou fenotípica, para (AOL), espessura de gordura subcutânea (EG) e espessura de gordura subcutânea na garupa (EGP8), em bovinos da raça Nelore, criados em pastagens tropicais sem complementação alimentar

\begin{tabular}{lcccccc}
\hline & AOL & EGS & EGP8 & MUSC & FRAME & PROF \\
\hline AOL & - & - & - & - & - & - \\
EGS & $0,05 \mathrm{~ns}$ & - & - & - & - & - \\
EGP8 & $0,04 \mathrm{~ns}$ & $0,68^{*}$ & - & - & - & - \\
MUSC & $0,15^{*}$ & $0,22^{*}$ & $0,11(\mathrm{~ns})$ & - & - & - \\
FRAME & $0,17^{*}$ & $0,07(\mathrm{~ns})$ & $-0,04(\mathrm{~ns})$ & $-0,49^{*}$ & - & - \\
PROF & $-0,09 \mathrm{~ns}$ & $0,24^{*}$ & $0,18^{*}$ & $0,57^{*}$ & $-0,48^{*}$ & - \\
\hline
\end{tabular}

ns $=$ não significativo. ${ }^{*}=$ significativo

Com o auxílio dos métodos utilizados para avaliação os programas ou as fazendas podem e devem alcançar seus objetivos destinados as melhorias de carcaça bovina na raça nelore, a qual foi referência para este estudo, alternando ambiente e capacidade de expressão genética gerando o fenótipo no qual mensuramos.

$\mathrm{Na}$ Tabela 4 tem-se a comparação dos manejos nutricionais antagonicamente utilizados, porém dentro de cada grupo de contemporâneo identificamos indivíduos fenotipicamente superiores devido a seu fator genético já que o ambiente teve seu valor igualado a todos. Esses indivíduos são desejados em qualquer manejo nutricional, lembrando que ele irá responder geneticamente em seu ambiente apropriado, eles serão utilizados em maior escalar na tentativa de transmitir suas características almejadas as progênies e com o passar do tempo ao rebanho comercial.

Tabela 3. Estimativa de correlação, simples ou fenotípica, para área de olho de lombo (AOL), espessura de gordura subcutânea (EG) e espessura de gordura subcutânea na garupa (EGP8), em bovinos da raça nelore, criados em pastagens tropicais com complementação alimentar

\begin{tabular}{lllllll}
\hline & AOL & EGS & EGP8 & MUSC & FRAME & PROF \\
\hline AOL & - & - & - & - & - & - \\
EGS & $0,12(\mathrm{~ns})$ & - & - & - & - & - \\
EGP8 & $0,23^{*}$ & $0,60^{*}$ & - & - & - & - \\
MUSC & $0,30^{*}$ & $0,24^{*}$ & $0,20^{*}$ & - & - & - \\
FRAME & $-0,06(\mathrm{~ns})$ & $0,01(\mathrm{~ns})$ & $0,03(\mathrm{~ns})$ & $-0,39^{*}$ & - & - \\
PROF & $0,17^{*}$ & $0,24^{*}$ & $0,14(\mathrm{~ns})$ & $0,48^{*}$ & $-0,30^{*}$ & - \\
\hline
\end{tabular}

ns = não significativo. ${ }^{*}=$ significativo

Tabela 4. Comparação de médias para área de olho de lombo (AOL), espessura de gordura subcutânea (EG) e espessura de gordura subcutânea na garupa (EGP8), em bovinos da raça nelore, criados em pastagens tropicais e pastagens tropicais com complementação alimentar

\begin{tabular}{lcc}
\hline & Manejo 1 & Manejo 2 \\
\hline AOL & $57,72 \pm 5,59^{*}$ & $69,75 \pm 6,35^{*}$ \\
EGS & $1,81 \pm 0,58^{*}$ & $2,68 \pm 0,95^{*}$ \\
EGP8 & $2,36 \pm 0,86^{*}$ & $3,61 \pm 1,32^{*}$ \\
\hline
\end{tabular}

\section{Conclusão}

A diferença entre os lotes separados por manejo alimentar é significativa para as estimativas. Porém, excluindo esse fator, as características avaliadas neste estudo contribuem significativamente para o melhoramento genético de carcaça, a escolha entre avaliação visual. No entanto, a avaliação por imagens de ultrassonografia dependerá da metodologia do programa escolhido, por 
ideologia própria do criador e pelos custos de cada uma, tanto AOL, EGS, EGP; enquanto que a musculosidade, tamanho e profundidade possuem correlações, portanto responderam sim a seleção promovendo mudanças e progressos genéticos, selecionando uma delas consequentemente estará melhorando as outras.

\section{Referências Bibliográficas}

Cancian, P. H., Gomes, R. C., Manicardi, F. R., Ianni, A. C., Bonin, M. d. N. \& Leme, P. R. 2014. Correlations of visual scores, carcass traits, feed efficiency and retail product yield in Nellore cattle. Scientia Agricola, 71, 17-22.

Faria, C. U., Andrade, W., Pereira, C. F., Silva, R. P. \& Lôbo, R. B. 2015. Bayesian analysis for carcass traits in Polled Nelore. Ciência Rural, 45, 317-322.

Faria, C. U., Magnabosco, C. d. U., Albuquerque, L. G. d., Bezerra, L. A. F. \& Lôbo, R. B. 2009. Estimativas de correlações genéticas entre escores visuais e características de carcaça medidas por ultrassonografia em bovinos Nelore utilizando modelos bayesianos linearlimiar. Revista Brasileira de Zootecnia, 38, 2144-2151.

Faria, C. U., Magnabosco, C. U., Reyes, A., Lôbo, R. B., Bezerra, L. A. F. \& Sainz, R.
D. 2007. Bayesian inference on field data for genetic parameters for some reproductive and related traits of Nellore cattle (Bos indicus). Genetics and Molecular Biology, 30, 343-348.

Koury Filho, W., Albuquerque, L. G., Alencar, M. M., Forni, S., Silva, J. A. \& Lôbo, R. B. 2009. Estimativas de herdabilidade e correlações para escores visuais, peso e altura ao sobreano em rebanhos da raça Nelore. Revista Brasileira de Zootecnia, 38, 2362-2367.

Yokoo, M. J. I., Werneck, J. N., Pereira, M. C., Albuquerque, L., Koury Filho, W., Sainz, R. D., Lobo, R. B. \& Araújo, F. 2009. Correlações genéticas entre escores visuais e características de carcaça medidas por ultrassom em bovinos de corte. Pesquisa Agropecuária Brasileira, 44, 197-202.

Article History:

Received 4 July 2017

Accepted 9 August 2017

Available on line 11 November 2017

License information: This is an open-access article distributed under the terms of the Creative Commons Attribution License 4.0, which permits unrestricted use, distribution, and reproduction in any medium, provided the original work is properly cited. 